\title{
Non-usages du téléphone portable : au-delà d'une opposition binaire usagers/non-usagers
}

Non-uses of the mobile telephone: beyond a binary opposition users/non users

\section{Thilo von Pape et Corinne Martin}

\section{Q OpenEdition}

1 Journals

\section{Édition électronique}

URL : http://journals.openedition.org/questionsdecommunication/416

DOI : 10.4000/questionsdecommunication.416

ISSN : 2259-8901

\section{Éditeur}

Presses universitaires de Lorraine

\section{Édition imprimée}

Date de publication : 1 décembre 2010

Pagination : 113

ISBN : 978-2-8143-0056-9

ISSN : 1633-5961

\section{Référence électronique}

Thilo von Pape et Corinne Martin, « Non-usages du téléphone portable : au-delà d'une opposition binaire usagers/non-usagers », Questions de communication [En ligne], 18 | 2010, mis en ligne le 01 décembre 2012, consulté le 19 avril 2019. URL : http://journals.openedition.org/ questionsdecommunication/416; DOI : 10.4000/questionsdecommunication.416 


\title{
NON-USAGES DU TÉLÉPHONE PORTABLE : AU-DELÀ D'UNE OPPOSITION BINAIRE USAGERS/NON-USAGERS
}

\begin{abstract}
Résumé. - Pour explorer les non-usages du téléphone portable, cet article se propose de relever deux défis. D'une part, il convient de sortir d'une perspective déficitaire des non-usagers; d'autre part, les auteurs entendent remplacer la dichotomie usage/nonusage en postulant au contraire l'existence d'une véritable continuité entre usage et non-usage. En effet, cet article montre que les différences entre les divers types d'usages (incluant l'usage limité ou partiel) sont bien souvent plus importantes que celles existant entre les usages et les non-usages. Ces résultats se fondent sur une comparaison de deux approches (quantitatives et qualitatives), deux échantillons et publics différents (allemand et français), issus de deux enquêtes différentes. Si le pari d'une telle comparaison est risqué - la confrontation souffrant de limites évidentes -, il n'en demeure pas moins qu'il permet aussi, in fine, de mieux comprendre l'usage.
\end{abstract}

Mots clés. - Non-usage, usage, usage partiel/limité, téléphone portable/mobile, approche quantitative, approche qualitative, Allemagne/France. 
$\square$

e téléphone portable occupe une place particulière parmi les technologies de l'information et de la communication (TIC). Alors que les sciences humaines et sociales (SHS) ont longtemps hésité à attribuer le statut de TIC au téléphone en général', l'arrivée du « portable » a considérablement augmenté l'intérêt des chercheurs pour ce dispositif. En une décennie à peine (à partir du milieu des années 90), il est devenu un objet banal, totalement intégré dans le quotidien des usagers. En France, ils sont près de 60 millions $^{2}$ (plus de $92 \%$ de la population) à l'utiliser et en Allemagne, l'autorité de régulation recense plus de 107 millions de lignes mobiles, soit 1,3 appareils par citoyen (Bundesnetzagentur, 2009). L'arrivée, au milieu des années 2000 , des portables de troisième génération permettant de surfer sur l'internet (le portable se transformant en objet de communication individuelle et de masse tout à la fois), mais aussi l'importance des enjeux économiques ${ }^{3}$ avec la fameuse question de la « convergence », ont contribué à asseoir la signification sociale du dispositif dans notre société, ce qui le rend particulièrement intéressant pour explorer le concept d'usage. Mais le téléphone portable constitue aussi une source riche pour explorer le non-usage des TIC. La littérature, encore limitée sur ce phénomène, concorde sur au moins deux enjeux pour mieux comprendre le non-usage. Le premier défi est de sortir d'une perspective déficitaire des non-usagers, d'accepter les légitimations qui peuvent les conduire à ne pas se servir d'une technologie, et d'analyser leur comportement de manière ciblée et rationnelle (« purposeful and rational »), comme le suggère Heinz Bonfadelli (2002 : 82). Conformément à ces enjeux, certains chercheurs ont opposé au concept des « have-nots », les « choose-nots » (Livingstone, Helsper, 2007) ou « want-nots » (Van Dijk, Hacker, 2003; Selwyn, 2003). II reste à explorer la nature des raisons poussant à un non-usage : s'agit-il d'un simple manque de besoin, d'une prise de position sociale, ou existe-t-il des raisons plus complexes et subtiles?

Le second défi pour la recherche sur les non-usages est de surmonter la simple dichotomie entre usage et non-usage. À cette fin, Sonia Livingstone et Ellen Helsper (2007 : 682) proposent d'établir un continuum, avec des gradations du non-usage en passant par l'usage réduit jusqu'à un usage plus fréquent («To map a continuum of use, with gradations from non-use, through low use to more frequent use »). Encore une fois, reste à explorer le potentiel de ce concept : les différents degrés d'intensité d'utilisation évoqués par Sonia Livingstone et Ellen Helsper ne

\footnotetext{
' Que ce soit le téléphone fixe ou les tout premiers téléphones portables au milieu des années 90, ces dispositifs ont d'abord suscité un manque d'intérêt par les sciences de l'information et de la communication (SIC) et par la sociologie en France (Flichy, 1992, 1997), mais aussi dans le monde anglo-saxon. Une autre explication à ce désintérêt résidait en son statut d'objet de communication interpersonnelle et non de masse.

2 Source Arcep (Autorité de régulation des communications électroniques et des postes). Accès : www.arcep.fr. Consulté en mars 2010.

3 Le volume d'affaires des seuls services mobiles (voix, sMs et services multimédias, dont accès à l'internet, etc.) a représenté en France en 2009 quelques 19, I milliards d'euros (Arcep, 2009) et en Allemagne en 200922 milliards d'euros (Bitkom, 2009).
} 
constituent pas le seul continuum qui lie usage et non-usage : les motifs d'utilisation, les normes sociales, le processus temporel de l'appropriation permettent aussi d'aller au-delà d'une simple opposition usage vs non-usage. La recherche sur les non-usages est freinée par un phénomène que l'on peut observer depuis longtemps dans la recherche sur les usages, à savoir la coexistence de deux écoles théoriques et méthodologiques qui communiquent peu entre elles alors qu'elles analysent les mêmes phénomènes: d'un côté, la recherche qualitative sur les usages, très répandue en France; de l'autre, la recherche quantitative sur l'adoption et les usages des innovations médiatiques, plus présente dans le monde anglophone. Par le biais de la confrontation entre ces deux courants, l'objectif de cet article est d'élargir les connaissances sur le non-usage du téléphone portable et ainsi de participer à l'analyse plus générale sur les non-usages des Tic. À cette fin, nous allons opposer dans la première section les deux courants théoriques et méthodologiques sur les usages et non-usages et introduire les deux études sur le non-usage du téléphone portable, issues de ces courants. Dans la deuxième section, nous nous concentrerons sur la manière de sortir d'une perspective déficitaire des non-usagers. La troisième section traitera de l'opposition entre usage et non-usage et permettra alors de définir différents continuums entre ces deux pôles au sein des échantillons étudiés.

\section{Une approche comparative}

Dans la diversité des approches qui aspirent à comprendre et expliquer les usages des TIC, « un courant spécifique de la sociologie des usages [...] s'est développé en France, en Belgique et au Québec » (Jouët, 2000 : 489), lequel se distingue des approches issues de la communauté scientifique anglo-saxonne, incluant aussi les pays qui participent intensément à l'échange scientifique de cette communauté à travers des publications anglophones, comme les pays de l'Europe du nord et de l'Asie. Alors que ces dernières se trouvent dans une grande continuité avec la recherche classique en communication de masse (" mass communication »), la sociologie des usages dans les pays francophones n'a pas évolué « dans le prolongement des études sur les usages des médias de masse » (ibid. : 49l). Cela concerne non seulement les théories appliquées, mais aussi les méthodes. Si la sociologie francophone des usages a longtemps privilégié les approches dites « qualitatives », les approches quantitatives sont beaucoup plus présentes dans le monde anglo-saxon. Une affirmation au fondement de cette contribution est que les deux approches sont complémentaires et non mutuellement exclusives et que l'avancement dans la recherche sur les usages - ainsi que sur les non-usages - passe par ce que Josiane Jouët (ibid. : 5|4) décrit comme « un dialogue plus étroit entre ces approches ». Nous allons donc appliquer ces deux approches à la question du non-usage du téléphone portable. La première s'inscrit dans la tradition francophone de la recherche sur les usages, la seconde prend comme point de départ la recherche sur la diffusion 
des innovations et démontre comment ses défauts peuvent être surmontés tout en sauvegardant une approche quantitative et standardisée. La base empirique de cette comparaison est constituée par deux enquêtes, l'une réalisée en France et l'autre en Allemagne; toutefois, cet article ne prétend pas s'étendre à une comparaison entre les non-usagers français ou allemands, mais se consacre uniquement aux approches théoriques et méthodologiques.

\section{Sociologie francophone des usages: la question du sens de l'usage}

La spécificité des études d'usage dans les pays francophones réside dans le fait qu'elles se sont d'emblée centrées sur les Tic en lien avec l'informatisation de la société. N'ayant pu bénéficier des apports des études de réception anglo-saxons, « en l'absence de théoriques et de modèles à appliquer, la sociologie des usages s'est donc forgée dans une effervescence de bricolage intellectuel et d'artisanat conceptuel » (ibid.: 493). Aussi s'est-elle constituée dans l'interdisciplinarité et s'est-elle inscrite dans le courant de l'autonomie sociale qui constituait de nouvelles approches sociologiques, tant dans le domaine de la famille que du travail. Les sociologues s'intéressent à la façon dont les individus s'approprient ces nouveauX outils que sont les TIC de façon subjective : à des fins d'émancipation personnelle ou professionnelle, à des fins de sociabilité. II importe donc de comprendre comment ils vont produire des normes pour légitimer leurs usages ou non-usages. D'une part, l'approche qualitative est inhérente à cette démarche, d'autre part, nous verrons combien l'hypothèse de la continuité entre les usages et les non-usages est heuristique.

\section{Un véritable continuum : des usages aux non-usages}

Pour Jacques Perriault (1989:218), la logique d'usage résulte d'une confrontation entre l'instrument, sa fonction et le projet de l'utilisateur. Si l'usage est rarement conforme à celui qu'avait strictement prescrit la sphère technicienne, c'est par la légitimation que se construit la norme: « Dans la constitution d'une norme intervient le facteur très important de la légitimation. C'est en quelque sorte la caution que l'usage est utile et licite ». Philippe Mallein et Yves Toussaint ( 1994) recourent pour leur part à la notion de signification sociale pour analyser le processus d'insertion sociale d'une TIC et son intégration ou non dans le quotidien des usagers. Josiane Jouët (2000: 50 I) évoque les « significations symboliques des objets de communication qui sont porteurs de représentations et de valeurs suscitant souvent l'adoption et la formation des premiers usages $\gg$. Si nous posons l'hypothèse qu'il existe une continuité entre les usages et les non-usages, nous pensons qu'il est tout aussi possible de mettre à jour les significations symboliques qui sont attachées au refus de l'usage et qui permettent aux non-usagers de 
se justifier. Le portable est incontestablement un succès et il semble bien que les individus qui refusent le portable développent une forme de résistance à cette pratique sociale normative qu'est devenu l'usage de cet objet, c'est ce que nous montrerons. Quant à Vincent Caradec (200 I), il analyse le non-usage des technologies chez des personnes âgées. La démarche est intéressante parce qu'une tendance domine; elle consiste à supposer que ces personnes âgées font preuve d'une véritable inaptitude:

\begin{abstract}
«Ce type d'explication repose sur l'idée qu'il existe une logique dominante (celle des classes supérieures ou celle de la diffusion de l'innovation) qui s'impose à tous et que les écarts observés par rapport à cette logique ne peuvent renvoyer qu'à une incapacité à s'y conformer. Un tel mode explicatif se refuse donc à envisager que ces écarts puissent être dotés de sens pour les acteurs concernés » (ibid. : 120).
\end{abstract}

L'auteur poursuit en développant l'hypothèse de la rationalité des usages et des non-usages:

« Nous considérons que les personnes rencontrées ont de bonnes raisons [c'est l'auteur qui met en italiques] d'avoir recours aux appareils techniques qu'elles utilisent et qu'elles ont aussi de bonnes raisons de ne pas avoir recours aux appareils qu'elles n'utilisent pas. Par bonnes raisons, nous entendons qu'elles sont capables de tenir un discours justifiant le fait qu'elles sont équipées (ou qu'elles ne le sont pas) et qu'elles utilisent (ou qu'elles n'utilisent pas) les différents objets technologiques [...] c'est ce que nous proposons d'appeler les "logiques d'usage" » (ibid. : । 2 | 122).

Vincent Caradec identifie ainsi quatre catégories d'usage : la logique utilitaire qui consiste à porter une appréciation sur l'utilité de l'objet considérée; la logique identitaire qui permet d'expliquer l'usage/non-usage par l'adéquation/ inadéquation de l'objet avec ce que l'on est; la logique de la médiation au travers de laquelle l'usage ou le non-usage est explicité par l'intervention d'un tiers; enfin, la logique d'évaluation qui consiste à porter un jugement sur l'objet technologique lui-même, ses caractéristiques, ses performances, en lui associant une image positive ou négative. II est entendu que ces quatre logiques vont bien souvent se combiner entre elles, aboutissant à la production de discours dits mixtes chez les usagers/non-usagers. Cette notion de logique d'usage peut être rapprochée de celle développée par Jacques Perriault (1989), exposée précédemment, en ce qu'elle renvoie à l'ensemble des normes et légitimations, justifications produites par les usagers et les non-usagers et nous ferons référence à ces bonnes raisons que mentionne Vincent Caradec. Nous évoquerons la notion de rationalité d'usage mais il importe de préciser qu'il n'est pas question pour nous d'inscrire cette notion de rationalité dans l'opposition épistémologique Raymond Boudon/Pierre Bourdieu, qui dépasserait largement le cadre de cet article. De même, la notion de rationalité que nous entendons développer n'a rien à voir avec celle des économistes (homo œconomicus), dès lors que nous reconnaissons plus volontiers chez l'humain la présence d'un inconscient de type freudien que celle d'une rationalité pure, quasi mathématique. La rationalité d'usage - telle que nous l'entendons - tend à montrer combien, face à une offre commerciale de plus en plus pléthorique (disponibilité grandissante de divers 
dispositifs techniques), les individus sont confrontés à la nécessité de faire des choix, de réaliser de véritables compromis. Ces compromis résultent de la prise en considération d'un ensemble de critères économiques, techniques, culturels, sociaux, ergonomiques, mais aussi liés à l'histoire personnelle des sujets : c'est ainsi que vont se mettre en place de véritables combinatoires d'usage, dès lors qu'un nouveau dispositif ne saurait remplacer intégralement et définitivement un ancien dispositif utilisé. En effet, Josiane Jouët (2000 : 500) a montré combien il convient de prendre en compte la généalogie des usages, dès lors « qu'il n'existe pas d'usage sui generis et que l'adoption des technologies de l'information et de la communication s'articule autour de techniques et de pratiques antérieures $\gg$. De même, Gilles Pronovost (1994 : 382) évoque la notion de continuum d'usage, en la renvoyant à deux aspects :

« On peut entrevoir que les usages sociaux de médias demandent à être insérés dans un continuum empirique, renvoyant non seulement à une diversité d'objets - téléviseur, magnétoscope, décodeurs sophistiqués (prescrivant eux-mêmes des modalités spécifiques d'appropriation) - mais également à un continuum historique faisant en sorte qu'un usage structuré, par exemple, en vienne à être déstabilisé, puis recomposé sous d'autres formes ».

C'est pourquoi nous posons l'hypothèse qu'il existe une continuité entre les légitimations des usagers et celles des non-usagers pour le téléphone portable et donc, d'une certaine manière, un continuum entre les usages et non-usages, dès lors que refuser le téléphone portable suppose que l'on ait fait le choix d'autres objets de communication, complémentaires, tels le téléphone fixe, les messageries sur l'internet, etc.; un choix fait y compris dans le temps, en lien avec son histoire personnelle, son mode de vie (choix qui pourra ainsi être remis en cause avec l'évolution du mode de vie). C'est ce que nous tenterons de démontrer.

La méthodologie retenue pour cette étude réalisée en France est volontairement de type qualitatif. Afin d'appréhender ces significations sociales, justifications produites par les usagers/non-usagers, les normes et logiques d'usages/nonusages qu'ils mettent en œuvre, nous avons fait le choix d'écouter les discours qu'ils produisent à cet égard. C'est pourquoi nous avons retenu la méthode de l'entretien. En effet, « l'entretien, qui va à la recherche des questions des acteurs eux-mêmes, fait appel au point de vue de l'acteur et donne à son expérience vécue, à sa logique, à sa rationalité, une place de premier plan » (Blanchet, Gotman, 1992 : 23). Ces deux auteurs poursuivent pour justifier l'enquête par entretien :

« [II] est ainsi particulièrement pertinente lorsque l'on veut analyser le sens que les acteurs donnent à leurs pratiques, aux événements dont ils ont pu être les témoins actifs; lorsque l'on veut mettre en évidence les systèmes de valeurs et les repères normatifs à partir desquels ils s'orientent et se déterminent. Elle aura pour spécificité de rapporter les idées à l'expérience du sujet. Elle donne accès à des idées incarnées, et non pas préfabriquées, à ce qui constitue les idées en croyances et qui, pour cette raison, sera doté d'une certaine stabilité » (ibid. : 27). 
Une première enquête par entretiens a donc été réalisée en 2003-2004; elle portait sur les usages/non-usages du téléphone portable chez les jeunes adolescents et leur famille, afin de cerner les représentations sociales que les individus se constituent de cet objet de communication. L'échantillon était constitué de 20 familles dont 3 familles de non-usagers ${ }^{4}$. Des entretiens semidirectifs ont été conduits séparément avec le jeune adolescent (en $3^{e}$ de collège et $2^{\text {nde }}$ de lycée) puis avec chacun de ses parents 5 . Une autre enquête, complémentaire, a été réalisée en 2008 sur les usages/non-usages de la photo et de la vidéo sur téléphone portable (en comparaison avec celles réalisées ou non avec un appareil photo numérique) auprès d'un échantillon de 12 étudiants de niveau 3 de Licence information-communication 6 . L'approche francophone de la première enquête ayant été présentée, il convient d'aborder les enjeux de la seconde approche quantitative des non-usages, de tradition anglo-saxonne, laquelle a servi de cadre théorique et méthodologique à la seconde enquête réalisée en Allemagne.

\footnotetext{
${ }^{4}$ Soit au total 9 non-usagers, ce qui constitue un très petit échantillon, et une faiblesse incontestable dans cette étude. Mais précisions deux points. D'une part, trouver des familles d'adolescents sans aucun portable relève de la gageure, eu égard au taux d'équipement des Français. De son côté, $G$. Gaglio (2005), dans son travail de thèse sur les usages du téléphone mobile, avait réussi à recueillir 12 entretiens avec des individus réfractaires (et non des familles) sur un total de 103 entretiens. Second point, d'autres enquêtes réalisées par nos soins, lesquelles s'intéressent aux usages limités de certaines fonctionnalités (photos, internet mobile) constituent une approche complémentaire que nous réutilisons dans cet article, dès lors que nous avons aussi fait l'hypothèse d'une absence de véritable rupture entre le non-usage et l'usage limité.

${ }^{5}$ Les entretiens approfondis ont été réalisés dans le centre de documentation du collège/lycée pour les adolescents. Que les proviseurs soient ici remerciés pour nous avoir ouvert la porte de leur établissement, tout en nous laissant œuvrer en toute indépendance. Quant aux parents, ils ont été interviewés séparément à leur domicile (ils étaient contactés après « accord 》 de l'adolescent). Chacun de l'ensemble de ces entretiens a duré d'une heure à une heure trente et a été intégralement retranscrit pour une analyse de contenu thématique.

${ }^{6}$ Une première partie de cette enquête consistait en un questionnaire passé auprès d'un échantillon de 247 étudiants issus de différentes formations : I ${ }^{\mathrm{e}}$ et $2^{\mathrm{e}}$ année de DUT Techniques de commercialisation et $L 3$ information-communication. Le questionnaire était composé d'environ 25 questions visant à caractériser l'usage (d'un point de vue quantitatif - quand, combien, avec qui, etc. -, mais aussi d'un point de vue qualitatif, par des descriptions des photos préférées, etc.). Puis 12 étudiants issus de la Licence information-communication ont été retenus, en vue d'obtenir un échantillon qualitatif « varié », comprenant des étudiants n'utilisant pas du tout leur téléphone portable pour les photos à ceux l'utilisant peu, en complément de leur appareil photo numérique (seuls deux étudiants de cet échantillon qualitatif n'avaient pas d'appareil photo numérique et utilisaient exclusivement leur portable, mais pas forcément de manière intensive). Les entretiens approfondis (une heure à une heure trente) ont été réalisés dans une salle de travail de la bibliothèque universitaire et ont été également intégralement retranscrits, en vue d'une analyse de contenu thématique.
} 


\section{Tradition anglo-saxonne : des modèles confrontés à la réalité empirique}

Étant donnée la diversité de la communauté scientifique anglo-saxonne en sciences de la communication, notre ambition se limite ici à présenter un courant. Ce courant ne se caractérise pas par un nom ou une école de chercheurs, mais par un objectif: trouver des modèles théoriques sur l'adoption, les usages et les non-usages des innovations, lesquels modèles peuvent être confrontés à la réalité empirique et améliorés en fonction des résultats. Sa base épistémologique est le « rationalisme critique » (Popper, 1973) selon lequel la communauté scientifique fait avancer les connaissances en testant des hypothèses et en améliorant les modèles théoriques en fonction des résultats de ces tests. Nous allons ainsi démontrer comment ce courant a engendré des concepts de plus en plus différenciés pour étudier usage et non-usage des innovations. Enfin, nous présenterons un modèle de l'appropriation du téléphone portable, qui a servi de base théorique à l'enquête présentée.

\section{Surmonter de façon constructive les limites de la théorie de la diffusion}

Everett Rogers (1962), le fondateur de la « théorie de la diffusion », peut être considéré comme un pionnier de cette tradition en ce qui concerne la question du non-usage des nouveaux médias. C'est dans les années 60 qu'il a résumé les différents approches sur la diffusion des innovations pour les intégrer au sein de la jeune discipline appelée «mass communication 》 (Katz, 1999: 145-146; Dearing, Singhal, 2006 : 20). Celle-ci était alors le courant le plus dynamique de la recherche sur la communication humaine dans le monde anglo-saxon (voir Delia, 1987), et elle offrait des concepts pertinents pour comprendre l'adoption et le refus des innovations, comme celui des leaders d'opinion (Lazarsfeld et al., 1944). Très rapidement, la théorie de la diffusion est devenue une approche centrale au sein de la « mass communication », et l'une des approches qui a attiré l'attention d'autres disciplines, même si elle était en même temps très contestée. Sur ce point, la tradition anglo-saxonne diverge des sciences de l'information et de la communication (SIC) françaises. En France, le constat des limites de la théorie de la diffusion a coïncidé avec une inscription des études sur les usages des nouvelles technologies dans une approche qualitative (Flichy, 1995), et qui se distançait de la conception poppérienne d'une recherche empirique standardisée. Le rejet de cette approche se reflète aussi dans le fait que la notion de « théorie de la diffusion » est souvent utilisée en tant que synonyme de la notion de « diffusionnisme », comme s'il s'agissait d'un courant normatif, alors que la communauté scientifique anglo-saxonne distingue l'approche théorique d'une part et l'idéologie du diffusionnisme d'autre part. Au sein de la « mass communication », des chercheurs ont continué, selon la conception de l'essai/ 
erreur, à développer des modèles plus appropriés permettant de mieux expliquer les usages et non-usages, tout en restant fidèle à l'approche de base quantitative et standardisée. Dans la prochaine partie, seront présentées les modalités selon lesquelles la voie quantitative initiée par Everett Rogers - et abandonnée par la recherche francophone - peuvent conduire tout de même à une meilleure compréhension des usages et non-usages en surmontant les limites de la théorie de la diffusion.

\section{Vers un regard plus différencié du non-usage}

Selon Everett Rogers (1962), les facteurs qui influencent la décision d'utiliser une innovation sont liés à l'adopteur potentiel, au système social et à la compatibilité entre ces deux paramètres et l'innovation. Ainsi certaines caractéristiques d'un individu vont-elles augmenter la probabilité d'une adoption précoce, comme par exemple son niveau d'enseignement, son statut socio-économique, son cosmopolitisme, la diversité des médias qu'il consomme (ibid.) ou encore son intégration dans des réseaux sociaux interpersonnels (Valente, 2006). Ces observations ont permis notamment d'expliquer les causes d'une « fracture numérique » au niveau des individus ou entre différentes sociétés (Rogers, 2000; Hargittai et al., 2002; Robinson, DiMaggio, Hargittai, 2003; Bonfadelli, 2002). Toutefois, les deux limites évoquées supra s'appliquent aussi à la théorie de la diffusion : premièrement, celle de la perspective déficitaire du non-usager, dénoncé comme « retardataire » face à une innovation souvent considérée comme a priori souhaitable (Rogers, 2003; Bonfadelli 2002; Selwyn, 2003); deuxièmement, celle du manque de différenciation dans la dichotomie entre l'usage et le non-usage, lié à la logique binaire adoption vs refus au cœur de la théorie de la diffusion. Pour considérer au mieux les raisons des non-usagers, on peut s'appuyer sur la théorie du comportement planifié (Theory of Planned Behavior, TPB, Ajzen, 1985), qui décrit les raisons d'adopter un certain comportement comme le produit d'un calcul intégrant des paramètres qu'on peut résumer en trois catégories, à savoir les conséquences personnelles de l'usage, les normes concernant l'usage et les restrictions et ressources facilitant l'usage. Cette théorie permet alors de considérer la décision de ne pas adopter une innovation comme un choix rationnel et de surmonter ainsi la perspective déficitaire des réfractaires. II ne s'agit pas d'un pur homo œeconomicus, car il suit les normes de son environnement. Cette approche a conduit à des études sur l'adoption de diverses technologies telles que le téléphone portable (Schenk, Dahm, Sonje, 1997) et différents services en ligne comme le commerce électronique (Hung, Ku, Chan, 2005) et les téléphones portables qui permettent d'aller sur l'internet (Teo, Pok, 2003). Reste alors à mieux appréhender la dichotomie entre usage et non-usage. Deux approches permettent de différencier davantage ces phénomènes : d'un coté, l'approche « des usages et gratifications » et, de l'autre, l'approche de la domestication. L'approche des usages et gratifications a été conçue à l'époque 
de la radio et de la télévision (Katz, Foulkes, 1962) et a vécu, selon Thomas Ruggiero (2000), une véritable renaissance ces dernières années dans l'analyse des usages des TIC (Leung, Wei, 2000; Sherry et al., 2006; Wei, Lo, 2006). Partant du principe que les médias constituent des alternatives fonctionnelles pour les usagers, cette approche est appliquée pour expliquer le succès ou l'échec de certains médias auprès des usagers, tels que le courrier électronique (Dimmick, 2000) ou le téléphone portable (Leung,Wei, 2000). En intégrant différents motifs d'utilisation (pragmatiques, sociaux, symboliques), cette approche propose des indices pour déterminer des logiques d'usage (Perriault, 1989; Caradec, 200 I). L'approche de la « domestication », développée par Roger Silverstone et Leslie Haddon (1996), reprend l'idée d'une appropriation active et constructive des consommateurs (Hall, 1980; de Certeau, 1980) pour analyser la manière dont ils « domestiquent » la technologie « sauvage » en l'introduisant dans leur espace et leurs routines. Cette approche a notamment été appliquée aux téléphones portables (Haddon, 2003), à l'ordinateur domestique (Bakardjeva, Smith, 200 I), à la vidéo à la demande (Ling, Nilsen, Granhaug, 1999) et à un certain nombre d'autres technologies domestiques (Lehtonen, 2003; Haddon, 2006)?

\section{Un modèle et une échelle pour mesurer les usages et non-usages du téléphone portable}

L'objectif de ce modèle est de surmonter les limites de la théorie de la diffusion sans rompre avec la recherche quantitative et standardisée. Plusieurs études mesurent des patterns d'appropriation de médias mobiles de façon quantitative, sur la base de sondages (Scott, 2003 - s'appuie sur le modèle d'influence sociale) ou d'observations (Hampton, Livio, Sessions, 20 I 0; Quandt, von Pape, 2010 - les auteurs s'appuient sur l'approche de la domestication). Ici sera utilisé un modèle qui réunit les différentes approches décrites dans cette section et les applique au téléphone portable : le «modèle de l'appropriation du téléphone portable » (Mobile Phone Appropriation Model, MPA, Wirth, Von Pape, Karnowski, 2008). En résumant les éléments principaux de la théorie de la diffusion, du modèle du comportement planifié, de l'approche des usages et gratifications et de l'approche de la domestication, ce modèle explique comment différents patterns d'utilisation du téléphone portable par un individu résultent d'un processus d'appropriation personnelle et sociale de l'innovation, lequel est déterminé par les gratifications recherchées, les normes en vigueur et les ressources et restrictions ressenties

\footnotetext{
7 Un grand nombre d'autres approches proposent aussi de considérer de façon plus différenciée le rôle des usagers mêmes dans la construction sociale de la technologie, comme par exemple l'approche de la « Social Construction of Technology » (scot) (Bijker, Hughes, Pinch, 1987, Fulk, 1993) et celle de la « Frame Analysis » (Goffman, 1974). Cependant, nous nous concentrons ici sur la domestication car cette approche s'inscrit dans une tradition de recherche sur les médias de masse, et elle est la plus répandue aujourd'hui dans la communauté scientifique anglophone, notamment dans les études sur le téléphone portable (Haddon, 2003).
} 
par les usagers. Conçu à l'origine pour analyser l'appropriation par les usagers, le modèle MPA sera ici appliqué au non-usage du téléphone portable. Le nonusage n'est certes pas une forme d'appropriation, mais, à partir du moment où l'innovation est suffisamment présente dans une société, il se constitue par des processus parfois analogues à ce qui se passe au cours de l'appropriation. Cette analogie a déjà été mise en avant par Richard Ling (1999). En effet, ce dernier a découvert des processus similaires entre les usagers et les non-usagers du téléphone portable au niveau de l'accumulation des connaissances et de la construction des attitudes sur le téléphone portable - même si l'usage reste évidemment exclusif aux usagers. Avec la croissance du nombre d'utilisateurs depuis 1999, les non-usagers sont de plus en plus souvent confrontés à la technologie (dans la rue, dans leurs familles, etc.). II leur est donc impossible d'ignorer complètement cette technologie. Cet état de fait implique alors de prendre en considération toute relation symbolique ou pragmatique avec une technologie, soit au cours d'une appropriation ou au cours d'un renoncement: s'en servir même de la façon la plus limitée, mais aussi en parler, et s'en faire des idées ou se construire une image symbolique. Pour mesurer et comparer les formes d'appropriation ou de renoncement, il faut un instrument empirique standardisé et quantitatif et en même temps très nuancé. À cette fin, une échelle de l'appropriation du téléphone portable (Mobile Phone Appropriation Scale) a été développée et appliquée dans plusieurs études (Von Pape, Karnowski, Wirth, 2008). Cette échelle représente en items, dans un questionnaire, différentes gratifications, des usages, normes et restrictions ainsi que différentes formes de communication sur le téléphone portable. La formulation de chacun de ces items est fondée partiellement sur des items utilisés dans d'autres études quantitatives (Leung, Wei, 2000; Wei, Lo, 2006; Trepte, Ranné, Becker, 2003) et partiellement sur des expressions enregistrées au cours d'entretiens semi-directifs avec des usagers et non-usagers du téléphone portable (Von Pape et al. 2006; Karnowski, Von Pape, 2008).

Dans l'étude quantitative, nous avons analysé l'appropriation du téléphone portable auprès des élèves de 16 classes de collège en Allemagne. || s'agit d'une étude de panel avec deux vagues qui ont permis de suivre l'évolution de l'appropriation. Pendant la lère vague (mars 2006), les élèves étaient en $7^{\mathrm{e}}$ et $9^{\mathrm{e}}$ classes (ils sont âgés de 13 et 15 ans environ), pendant la $2^{\mathrm{e}}$ vague (janvier 2007), ces mêmes élèves étaient en $8^{\mathrm{e}}$ et $10^{\mathrm{e}}$ classes (Von Pape, 2008). Dans l'ensemble, $86 \%$ des élèves ont participé à l'enquête, $14 \%$ étaient soit absents le jour de l'enquête, soit n'avaient pas obtenu l'autorisation de leur parents. 86 \% des élèves ayant participé à la Ière vague ont aussi participé à la $2^{\mathrm{e}}$ vague (mortalité

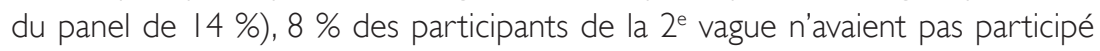
à la première. Parmi les participants de la lère vague, nous avons compté 52 non-usagers ( $13 \%$ ) et 345 usagers, et parmi ceux de la $2^{\mathrm{e}}$ vague, 34 non-usagers (9\%) et 330 usagers. 


\section{Confrontation des deux approches et enquêtes}

Le pari de confronter ces deux approches et enquêtes est ambitieux et risqué. En ce qui concerne la démarche qualitative, elle cherche à comprendre et mettre à jour une logique personnelle d'usage/non-usage en retraçant l'histoire singulière du sujet. Mais la tentative de généralisation, par la mise en évidence de typologies ou répertoires d'usage, rencontre forcément des limites. Dans l'approche quantitative, les mesures standardisées permettent de faire la comparaison, favorisant ainsi les possibilités de généralisation. Néanmoins, la volonté demeure de ne pas glisser dans la réduction et la simplification, afin de toujours pouvoir rendre compte de la complexité de l'appropriation comme caractéristique propre à chaque individu. Si les différences entre ces deux approches demeurent irréductibles, en revanche, les concepts proposés par les deux courants pour mieux comprendre et différencier le non-usage paraissent très proches. C'est le cas notamment des propositions pour étudier un continuum d'usage, déjà évoqué par Sonia Livingstone et Ellen Helsper (2007) : le concept utilisé par Gilles Pronovost (1994) utilise le même terme de continuum, et le modèle MPA permet d'identifier des formes d'entrée en relation avec le téléphone portable qui jouent un rôle dans l'appropriation autant que dans le renoncement de la technologie (par exemple, se faire une opinion sur les gratifications et les normes). Comment ces différences et complémentarités entre les deux approches se manifestent-elles dans le cadre d'enquêtes empiriques sur le nonusage du téléphone portable? Et quels sont les gains à tirer de cette démarche de comparaison, notamment pour sortir de la perspective déficitaire du non-usager et pour aller au-delà de la simple opposition entre usager et non-usager? Les deux prochaines parties répondront à ces questions en opposant et synthétisant les résultats des enquêtes.

\section{Vers une meilleure compréhension des non-usagers}

\section{Refus ou apostasie du portable}

Dans l'approche qualitative, nous nous intéressons au non-usage chez les adultes ${ }^{8}$, lesquels refusent de s'équiper en téléphone portable. L'ouvrage L'apostasie de la télévision, d'Élisabeth Castro-Thomasset (2000) apporte un éclairage intéressant sur le non-usage d'un média, en l'occurrence la télévision. L'auteure a fait le choix d'« appréhender un objet à travers ce qu'en livrent ses non-usagers » (ibid. : 13) et montrer ainsi la pertinence à concevoir les non-usagers comme une source d'information utile à l'analyse sociologique. « Le terme « apostasie » est utilisé aujourd'hui dans la sphère politique ou religieuse; il désigne le fait

${ }^{8}$ Ces adultes font partie des familles d'adolescents sans portable qui ont été interviewés en 20032004 (Martin, 2007b). 
de renoncer à son parti politique ou à sa religion pour un ou une autre, et ce, publiquement » (ibid. : | 3). En effet, il est apparu très tôt que si les individus n'avaient pas de portable, cela n'était pas tant la conséquence d'une impossibilité, matérielle par exemple, encore moins celle d'une incapacité à s'adapter à l'objet technique, mais que cela résultait plutôt d'un choix et donc d'un refus clairement affirmé et assumé. « Rejeter un objet que tout le monde possède, c'est-à-dire qui s'inscrit dans une pratique sociale normative, signifie qu'en optant pour la rupture, on tente d'échapper à la pression sociale d'une attitude conformiste » (ibid. : 21). L'auteur considère que l'on peut qualifier d'apostats les individus qui rejettent l'objet télévision dès lors que plus de $90 \%$ des familles en sont équipées. Nous suivrons le raisonnement de l'auteure, puisque le taux d'équipement du téléphone portable dépasse les $90 \%$. C'est pourquoi il nous a semblé qu'avoir un portable peut, de nos jours, être assimilé à une pratique sociale normative. À leur façon, ces non-usagers résistent à la norme en refusant l'usage. Si l'on revient sur les études qualitatives d'usage du téléphone portable en France, il est incontestable qu'elles sont nombreuses et se sont inscrites dans des problématiques centrées sur le lien social, que ce soit à travers l'analyse des usages voix/SMS (Licoppe, 2002; Rivière, 2002; Réseaux « Mobiles 》, 2002; Jauréguiberry, 2003), des photos/vidéos (Jarrigeon A., Menrath J., 2007; Réseaux « Les usages avancés du téléphone mobile », 2009) ou bien encore de l'internet mobile (Réseaux « Les usages avancés du téléphone mobile », 2009). Elles ont aussi traité de la question centrale du portable comme objet personnel et de l'autonomie qu'il favorisait (Réseaux « Mobiles », 2002; Martin, 2007b) avec son pendant, le rapport au pouvoir (Réseaux « Mobiles », 2002; Jauréguiberry, 2003) ainsi que la généralisation de l'urgence (Jauréguiberry, 2007). Mais il faut avouer que bien peu d'études se sont centrées, voire ont abordé seulement la question du non-usage. Gérald Gaglio (2005) analyse les usages dans une perspective intéressante qui prend en considération la dimension de consommation et son travail fait partie des rares travaux qui s'intéressent aux non-usagers du téléphone mobile. Ainsi évoque-t-il les réfractaires, pour montrer combien il faut effectivement aller au-delà de la simple absence de besoin. II recourt à la notion de rationalité en finalité, mais ajoute aussi qu'elle « se complète d'une rationalité touchant aux valeurs des individus, et ce concernant, par exemple, des domaines cadrant l'existence sociale comme le travail et le couple. [...] L'efficacité, en tant qu'objectif, est parfois laissée de côté au profit d'une critique de principes et de l'affirmation de conceptions qui surplombent la décision de ne pas s'équiper d'un téléphone portable » (ibid. : | 82-183). Dans notre enquête, le discours des nonusagers se situe dans le champ des valeurs, il est même idéologique et constitue une véritable posture critique. II est construit sur le mode d'une rhétorique, visant à se démarquer des autres. La critique de la société de consommation est, semble-t-il, le socle de l'argumentaire : « La consommation gratuite, c'est pas notre truc » dira M. Jérôme (48 ans, sans portable, instituteur, trois enfants dont le dernier en seconde, sans portable, conjointe et enfants sans portable) mais

\footnotetext{
${ }^{9}$ Voir supra, source Arcep. Accès : www.arcep.fr.
} 
l'analyse s'étend à une réflexion critique sur l'ensemble de la société, dans toutes ses dimensions, tant politiques, qu'économiques, sociales, environnementales, familiales et éducatives. C'est un discours engagé, presque militant, de dénonciation. Le téléphone portable a perdu toute dimension technique et n'est plus appréhendé que sous le seul angle d'objet de consommation: « Pour nous transformer en consommateurs, on n'existe plus en tant que personnes, vous n'êtes plus qu'un truc de profit quoi » s'insurge M. Petit (47 ans, sans portable, fonctionnaire, fils unique en seconde, sans portable, conjointe et enfant sans portable). Ces non-usagers évoquent une absence de «besoin », laquelle renvoie en réalité à une véritable inutilité sociale. II est intéressant de constater qu'il existe aussi une continuité entre les légitimations de non-usage avec les légitimations d'usage produites par les usagers ${ }^{10}$. En effet, les arguments sont quasi inversés: la joignabilité permanente, premier argument des usagers, est considérée néfaste, liée au refus de se soumettre à la densification du temps que favorise l'outil, refus que pratique, non sans humour, Mme Petit (46 ans, sans portable, conseillère principale d'éducation, fils unique en seconde sans portable, conjoint et enfant sans portable) :

« Moi, je rigole... je suis joignable 90 \% de mon temps, parce que j'ai une carte téléphonique, au bureau, j'ai le téléphone, à la maison, j'ai le téléphone et entre les deux, sur la route, c'est interdit de s'en servir alors... [rires] et pour les $10 \%$ restant, c'est mon temps pour moi... ».

Un autre argument des usagers, tout aussi essentiel - à savoir la réassurance offerte par le portable - est totalement déconstruit par M. Malet (44 ans, sans portable, cadre informaticien, trois enfants dont le deuxième en seconde, conjointe sans portable), en le réduisant au rang de « futilité » :

«Quand je réfléchis en tant qu'informaticien ou statisticien, je me rends compte que les voitures tombent de moins en moins souvent en panne et les crevaisons, ils vont enlever les roues de secours parce qu'il y a de moins en moins de crevaisons, alors quand j'entends des réflexions comme ça « il me faut à tout prix un portable parce que si je crève avec ma voiture, je téléphone à quelqu'un pour qu'il me répare » je trouve que c'est vraiment futile ».

Mais qu'on ne s'y trompe pas, ils ne sont en rien technophobes et sont à la pointe du progrès sur d'autres aspects (équipement informatique, internet, etc.). Pour sa part, Gérald Gaglio (2005) évoque les usages alternatifs des réfractaires qui utilisent donc d'autres modes de communication médiatisée que le téléphone portable. Nous conclurons qu'ils ont donc de bonnes raisons de ne pas utiliser le téléphone portable, lesquelles bonnes raisons constituent in fine une véritable résistance face à une pratique sociale normative.

L'explication mentionnée ci-dessus renvoie à la notion d'usage comme un construit social (Chambat, 1994) et démontre que le non-usage peut ainsi être compris comme un acte social. Toutefois, et comme le soulignent Philippe

10 II est intéressant de noter qu'ils ont tous « basculé » dans le changement et sont aujourd'hui équipés (recontactés en 2008), à la suite de l'évolution de leur mode de vie (par exemple : un enfant parti faire des études dans une autre ville, etc.). 
Breton et Serge Proulx (2006 : 255), un usage peut aussi s'expliquer simplement « par le poids de contraintes externes [...] et par des caractéristiques propres à l'usager » comme notamment son capital économique et ses compétences techniques. Ces questions seront approfondies dans la section suivante à partir de l'enquête réalisée auprès de collégiens allemands.

\section{Différents patterns de non-usage}

La distinction entre des non-usagers involontaires des nouvelles technologies de la communication ( " have-nots ») et des non-usagers volontaires (" choosenots », Livingstone, Hesper, 2007; « want-nots », van Dijk, Hacker, 2003; Selwyn, 2003) constitue le point de départ de cette analyse. Notre échantillon comprend 52 non-usagers dans la $\left.\right|^{\text {ère }}$ vague $(13 \%)$ et 34 dans la $2^{\text {e }}$ vague $(9 \%)$. Pour savoir dans quelle mesure le non-usage dans notre échantillon est volontaire, nous avons appliqué une échelle fondée sur trois items représentant différents indicateurs d'un non-usage volontaire, comme par exemple « Je ne regrette pas de ne pas avoir de téléphone portable $»^{\prime \prime}$. Le taux de ceux qui se déclarent comme plutôt involontaires (en dessus de 2,5 sur l'échelle qui va de I à 4) est de $58 \%$ en moyenne pour les deux vagues. Puisque l'autonomie d'un adolescent augmente normalement quand il grandit, on peut s'attendre à une diminution du non-usage non volontaire auprès des étudiants plus âgés. En comparant les étudiants plus jeunes avec les plus âgés, nous constatons effectivement que le non-usage volontaire augmente au cours de l'adolescence : pour les non-usagers des $7^{\text {e }}$ et $8^{\text {e }}$ classes (13 à 14 ans), seulement $34 \%$ se disent volontaires, mais ce taux passe à $81 \%$ pour les non-usagers de la $9^{e}$ et $10^{e}$ classes. Si l'usage du téléphone portable et des TIC en général reflète la croissante autonomie de l'adolescent (Martin, 2007b; Holmes, Russel, 1999), le non-usage le reflète également. En comparant les ressources des adolescents, on constate d'abord que les non-usagers involontaires ne disposent que de 16,02 $€$ d'argent de poche par mois en moyenne, alors que cette moyenne est de 23,35€ pour les non-usagers volontaires et $26,80 €$ pour les usagers. Par ailleurs, $44 \%$ des nonusagers involontaires affirment que leurs parents ne veulent pas qu'ils investissent beaucoup de temps et d'argent dans un téléphone portable, ils ne sont plus que 38 \% auprès des non-usagers volontaires. Même si ces différences ne se révèlent pas significatives au sein de notre petit échantillon, il apparaît cependant que les ressources financières et les restrictions parentales jouent un rôle dans le non-usage involontaire du téléphone portable. II reste alors à comprendre le comportement des non-usagers volontaires, qui auraient pourtant les moyens et la permission parentale de se servir de la technologie. Pour répondre à cette question, il faut se tourner vers les désirs et besoins de chacun (en

"Quatre options de réponse entre «Pas du tout d'accord » (I) et «Tout à fait d'accord » (4). La validité interne de cette échelle (alpha de Cronbach) est satisfaisante avec 0,80 pour la première vague et 0,72 pour la seconde vague. 
suivant l'approche individualiste des usages et gratifications) et vers les normes sociales auxquelles ils sont confrontés dans leur environnement quotidien (grâce notamment à la théorie du comportement planifié). Pour comparer les gratifications que les deux catégories de non-usagers ainsi que les usagers voient dans la technologie, nous nous fondons sur des échelles représentant quatre dimensions de gratifications : le prestige, le divertissement, la gestion des relations et la sécurité. Par exemple, un item pour la gestion des relations est: « Pour moi, un aspect important du téléphone portable est qu'il me permet de maintenir le contact avec mes amis $\rangle^{12}$. La comparaison entre les groupes de non-usagers (illustration I) montre que les non-usagers volontaires évaluent l'intérêt du téléphone portable comme moindre sur toutes ces dimensions ${ }^{13}$. En revanche, pour les non-usagers involontaires, la valeur de prestige est jugée comme extrêmement importante. Les usagers se trouvent entre les deux types de non-usagers dans leur évaluation de l'importance de la valeur de prestige, de la gestion des relations et du divertissement. Quant à la sécurité, elle est qualifiée de plus importante par les usagers: il s'agit d'ailleurs de la seule différence significative entre usagers et non-usagers. Ces chiffres soulignent le constat plus général de Neil Selwyn (2003 : I l0) que les interactions des individus avec les TIC sont plus complexes que la simple dichotomie entre « usager/non usager » reprise par une grande partie de la littérature existante. Ne pas considérer les non-usagers comme un groupe homogène s'avère essentiel pour l'interprétation des données présentées: une simple distinction binaire entre usagers et nonusagers aurait montré que les non-usagers cherchent à peu près les mêmes gratifications dans le téléphone portable que les usagers et aurait alors confirmé l'idée reçue selon laquelle l'utilisation ou non résulte des restrictions des nonusagers. La distinction entre non-usagers volontaires et involontaires démontre au contraire que les non-usagers volontaires divergent nettement des deux autres groupes : c'est un désintérêt dans une technologie superflue qui explique le non-usage volontaire, et non les restrictions.

\footnotetext{
12 Les dimensions avaient été identifiées lors d'autres études quantitatives et qualitatives (voir von Pape, Karnowski, Wirth, 2008). Pour le prestige, l'échelle sur la base de 4 items a une consistance

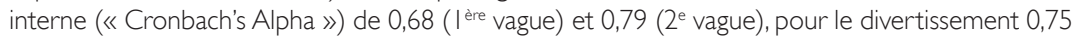
( I ère vague) et 0,84 ( $2^{\mathrm{e}}$ vague, 2 items), pour la gestion des relations 0,70 (I ère vague) et 0,74 ( $2^{\mathrm{e}}$ vague, 5 items), pour la sécurité un seul item a été utilisé. Pour des différenciations semblables, voir Leung, Wei, 2000; Wei, Lo, 2006; Trepte, Ranné, Becker, 2003). Pour les non-usagers, les questions ont été posées au conditionnel (« un aspect important du téléphone serait que...»).

${ }^{13}$ Les différences sont significatives pour les deux vagues au niveau du prestige $\left(T_{\text {vaguel }}=2,\left.6\right|^{*}\right.$; $\left.T_{\text {vague } 2}=3,22 * *\right)$, de la gestion des relations $\left(T_{\text {vaguel }}=3,51 * * ; T_{\text {vague } 2}=3,12 * *\right)$ et de la sécurité $\left(T_{\text {vaguel }}\right.$ $\left.=2,46 * ; T_{\text {vague } 2}=3,13^{* *}\right)$.
} 
Illustration I : Moyennes d'importance attribuée aux différentes gratifications (moyennes des valeurs des deux vagues; I =pas du tout important, 4=très important, moyenne des deux vagues).

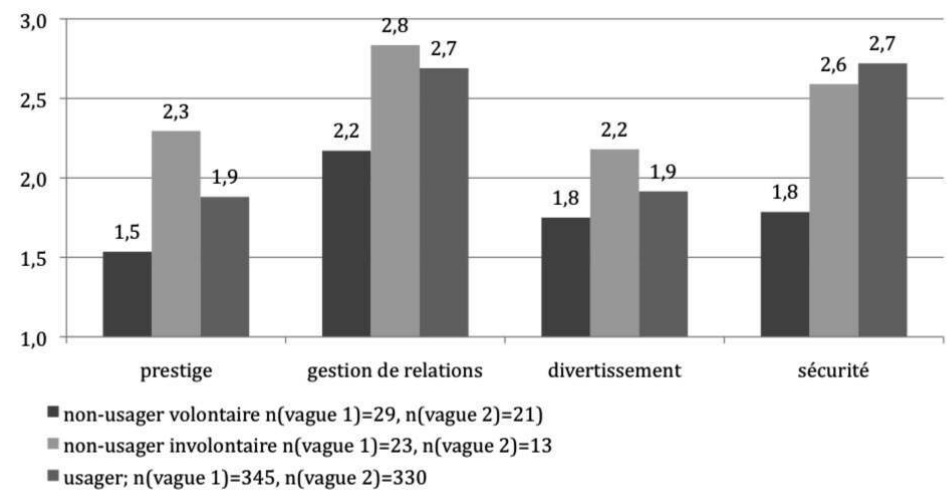

L'être humain n'agit pas seulement en fonction des intérêts individuels, mais aussi en fonction des normes sociales de son environnement (Ajzen, 1985). Par conséquent, la perspective individualiste des usages et gratifications (Elliot, 1974) doit être complétée par une prise en considération des normes relatives à l'utilisation du téléphone portable. Sur la base d'études qualitatives préliminaires (Von Pape et al. 2006; Karnowski, Von Pape, 2008), deux sortes de normes ont été identifiées comme déterminantes pour l'utilisation du téléphone portable: celles qui impliquent une utilisation discrète de la technologie et celles qui exigent d'être en permanence joignable ${ }^{14}$. Les premières compliquent souvent l'utilisation du téléphone portable alors que les secondes la favorisent. Ces normes peuventelles expliquer davantage la volonté de certains non-usagers de ne pas avoir de téléphone portable? Effectivement, on peut constater des perceptions inverses auprès des deux groupes de non-usagers : les normes sur la joignabilité sont perçues comme plus importantes par les non-usagers involontaires que par les non-usagers volontaires, alors que les normes sur la discrétion sont perçues comme plus importantes par les non-usagers volontaires (Illustration 2) ${ }^{15}$. Si les non-usagers volontaires ne veulent pas utiliser de téléphone portable, ce n'est donc pas, dans la plupart des cas, en opposition avec leur environnement personnel immédiat, mais bien en accord avec les personnes qui les entourent.

\footnotetext{
${ }^{14}$ L'échelle sur les normes de discrétion contient 5 items, comme par exemple « II est considéré comme impoli d'utiliser le téléphone portable quand on est en compagnie d'autres » (alpha de Cronbach $=0,66$ pour la lère vague et 0,72 pour la $2^{e}$ vague). L'échelle sur les normes de joignabilité contient 5 items, comme par exemple « Dans mon cercle d'amis, on a intérêt à avoir un téléphone portable si l'on veut participer » (alpha de Cronbach=0,55 pour la lère vague et 0,64 pour la $2^{\text {e }}$ vague).

${ }^{15}$ Ces différences sont significatives concernant la différence entre les groupes pour les normes de discrétion pour la lère vague $(T=2,69 *)$ et les normes de joignabilité pour la $2^{\text {de }}$ vague $(T=2,3 \mid *)$.
} 
Ce résultat ne contredit pas forcément l'hypothèse d'une apostasie du téléphone portable auprès de notre échantillon de non-usagers, car il se peut que les adolescents voient, au-delà de leur environnement social immédiat, une idéologie dominante du téléphone portable contre laquelle ils se révoltent. La conviction que le téléphone ne répond simplement pas aux besoins de l'individu et constitue une technologie « superflue » peut faire partie d'une telle révolte. II faut aussi comparer les non-usagers avec les usagers : ces derniers attribuent une importance égale aux deux formes de normes, mais à un niveau plus élevé que les non-usagers ${ }^{16}$. Ceci peut être expliqué par le fait que, en tant qu'usagers, ils sont plus souvent confrontés aux normes que les non-usagers (penser à toujours emporter le téléphone pour être joignable, penser à l'éteindre dans certains contextes pour être discret) (Illustration 4).

Illustration 2 : Perception des normes de discrétion et de joignabilité ( I = pas du tout important, 4 = très important).

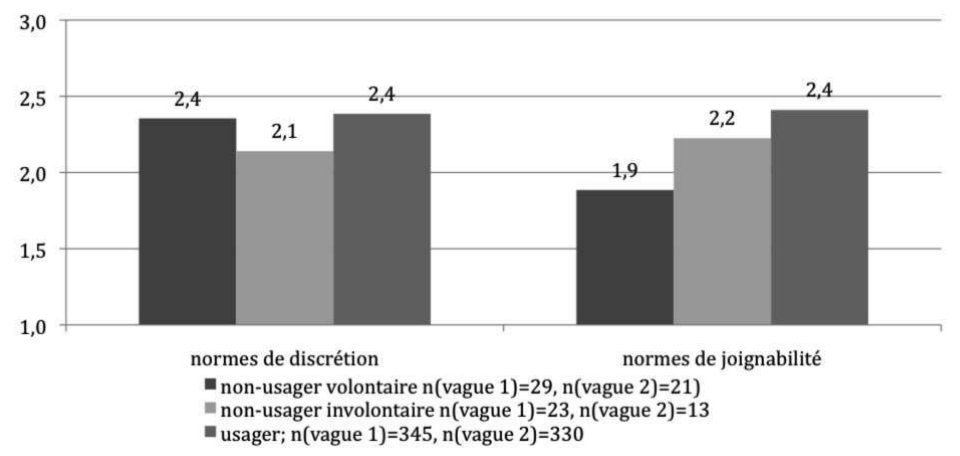

Nous avons démontré dans cette partie que les non-usagers ne forment pas un groupe homogène, mais qu'ils se distinguent par les motifs du non-usage en des non-usagers volontaires et involontaires. Cette distinction permet d'identifier, parmi les non-usagers volontaires, des raisons pour le non-usage qui vont au delà des visions déficitaires et idéologiques du non-usager. Ainsi les non-usagers volontaires de l'échantillon voient-ils clairement et à tout niveau un moindre intérêt dans le téléphone portable, et ils côtoient dans leur environnement proche des normes plutôt critiques vis-à-vis de cette technologie.

${ }_{16}$ Les différences sont significatives pour les normes de joignabilité pour les deux vagues $\left(T_{\text {vaguel }}\right.$ $=4,92 * * ; T_{\text {vague } 2}=4,18 * *$. 


\section{Confrontation : des logiques du non-usage}

L'objectif de cette première confrontation des résultats est d'affiner notre image des non-usagers au-delà des simples visions de « retardataires » limités dans leurs ressources et/ou irrationnels dans leur comportement. II faut pourtant commencer par une concession :certains individus dans l'échantillon des étudiants correspondent bien à cette vision déficitaire. En effet, certains d'entre eux sont des non-usagers malgré eux, en raison d'une absence d'autorisation parentale et/ ou de moyens financiers. On pourrait aussi parler d'« usagers empêchés », car au niveau des gratifications qu'ils attribuent au téléphone portable et au niveau de la pression sociale qu'ils ressentent à être joignable en permanence, ils sont plus proches des usagers que des non-usagers volontaires. Avec l'autonomie que les adolescents gagnent au fur et à mesure qu'ils avancent en âge, le phénomène d'un non-usage involontaire diminue. Cette observation peut expliquer pourquoi on ne trouve pas d'éléments reflétant une attitude involontaire dans les discours des adultes cités ci-dessus. Néanmoins, les adolescents ne sont pas les seuls susceptibles d'être des non-usagers involontaires: un manque de moyens financiers ou de compétences techniques peut aussi apparaître auprès des adultes, notamment les personnes âgées rencontrent des difficultés à adhérer à la nouvelle technologie (Renaud, van Biljon, 2008). Concentrons-nous à présent sur les non-usagers qui renoncent de façon volontaire au téléphone portable et qu'on trouve dans les deux études. L'analyse des gratifications potentielles qu'ils perçoivent dans le téléphone portable révèle qu'ils n'attribuent simplement pas d'importance aux promesses de divertissement, de prestige, de gestion des relations et de sécurité. De l'autre côté, leur environnement immédiat n'est pas non plus favorable à l'innovation: les normes prédominantes sont plutôt strictes vis-à-vis des gênes causées par le téléphone portable, et plutôt tolérantes concernant l'exigence d'être joignable. Toutefois, il faut souligner que l'appropriation n'a été étudiée ici que sous l'aspect des normes et des gratifications. De manière complémentaire, l'approche qualitative permet une perspective plus globale autorisant la prise en considération, pour les individus interrogés, d'un environnement plus large. À première vue, les affirmations des adultes correspondent aux résultats trouvés auprès des adolescents, comme par exemple M. Malet qui remet en question la gratification de sécurité du téléphone portable en cas de panne de voiture quand il affirme qu'il ne craint simplement pas un tel événement. Il apparaît pourtant que les non-usagers adultes produisent plutôt un véritable discours engagé contre le téléphone portable, contrairement au désintérêt évoqué dans l'approche quantitative. Et ce refus engagé, qui trouve son origine dans un rejet de la société de consommation (voir supra), va de pair avec un discours de déconstruction sociale de l'utilité de la technologie. En effet, les non-usagers reprennent les légitimations des usagers, mais pour mieux les tourner en dérision. C'est ainsi que la joignabilité, considérée comme essentielle pour les usagers, devient au contraire caduque, inutile chez les nonusagers. De même, la réassurance que conférerait, selon les usagers, le portable, est entièrement réduite à néant par les non-usagers. En ce sens, il est possible 
de parler d'une véritable continuité entre les légitimations d'usages et celles des non-usages. Ce constat s'inscrit dans une certaine continuité déjà révélée par les résultats quantitatifs (voir supra). En ce qui concerne les normes, les deux résultats apparaissent contradictoires. En effet, les non-usagers adultes se présentent comme une minorité d'apostats face à la norme sociale dominante associée au téléphone portable, alors que les non-usagers volontaires parmi les adolescents se disent en accord avec les normes dominantes, critiques visà-vis du téléphone portable, qu'ils côtoient dans leur environnement proche. Une explication de ces différences pourrait être liée aux différences entres les échantillons : d'un côté, une jeune génération plutôt conformiste et, de l'autre, des adultes rebelles. Mais un autre paramètre peut aussi être pris en compte: l'apostasie des adultes se réfère plutôt à leur positionnement face aux normes sociales dominantes, alors que l'évaluation par les jeunes des normes dominantes dans leur environnement concerne l'entourage personnel immédiat, c'est-à-dire les amis et la famille. Ces deux constats pourraient alors être conciliés avec l'hypothèse que l'environnement immédiat des non-usagers (adolescents et adultes) constitue un noyau critique dans une société dominée par l'emprise du téléphone portable. II manque les éléments empiriques pour tester cette hypothèse, mais la littérature sur les normes dans les réseaux sociaux confirme que les réfractaires se regroupent dans des petites cellules (Kincaid, 2007).

\section{Différenciations au sein du non-usage}

Entre autres distinctions, la dernière section a révélé que celle entre non-usagers volontaires et involontaires se révèle heuristique dans l'analyse du non-usage du téléphone portable auprès des adolescents : les différences au sein du groupe des non-usagers sont plus importantes que les différences entre l'ensemble des non-usagers et les usagers. II s'agit alors de remplacer l'opposition binaire entre usagers et non-usagers par une perspective plus nuancée qui prenne en compte la continuité entre les différentes formes d'usages/non-usages.

\section{Une véritable rationalité d'usage}

Dans l'approche qualitative des usages du téléphone portable, nous avons développé l'hypothèse de l'objet incorporél7 (Martin, 2007a) en ce que le téléphone constitue une véritable extension corporelle: il reste toujours

\footnotetext{
17 Lorsque J.-Cl. Kaufmann ( 1997) parle d'habitudes incorporées, il montre combien cette incorporation, cette familiarisation par le corps est éloignée de la pensée rationnelle et ne suscite donc aucune réflexivité. Nous nous référons aussi aux travaux sur la culture matérielle, qui montrent combien certains objets de petite taille peuvent être intégrés dans le schéma corporel (Julien, Warnier, 1999).
} 
à portée de main, une condition essentielle de son appropriation, de son intégration dans les routines corporelles. Or, cette intégration dans le quotidien ne rime pas toujours avec un usage important, elle est tout autant le fait des faibles, voire très faibles usagers pour lesquels le portable est néanmoins devenu tout aussi indispensable. Nous pourrions dire que ceux-ci pratiquent un usage partiel, limité. Dans le cadre de l'approche qualitative présentée (voir supra), une enquête complémentaire a été réalisée en France sur les usages de la photo et vidéo sur téléphone portable chez de jeunes adultes ( 12 interviewés, étudiants de $3^{e}$ année de licence information-communication), et nous avons émis l'hypothèse d'une véritable rationalité d'usage. En effet, face à l'importance de l'offre disponible (différents dispositifs : appareil photo numérique, téléphone portable faisant photo, lecteur musique, etc.), il semble que ces jeunes adultes mettent en œuvre une rationalité d'usage qui viserait à définir un compromis, une sorte d'orientation de la pratique, et qui résulterait de l'analyse personnelle de tout un ensemble de critères : d'abord la qualité des photos en fonction de ce que l'individu souhaite en faire (les conserver sur l'ordinateur ou non), mais aussi les contraintes matérielles liées à l'objet (taille de l'APN ${ }^{18}$ et possibilité ou non de l'avoir toujours sur soi' ${ }^{19}$ ), les contraintes techniques (compatibilité des formats avec l'ordinateur pour le stockage), les contraintes économiques (gratuité de l'email ou du postage sur msn/blogs vs coût du MMs; valeur économique du téléphone vs celle de l'APN), etc. Et cet arbitrage n'a rien d'absolu, il est au contraire relatif. Nous pourrions le qualifier de plastique, au sens où il ne constitue par une forme figée, mais va être refaçonné en permanence et ainsi redéfinir de nouvelles configurations d'usage en fonction des autres appareils disponibles, conduisant à un réexamen des critères. Comme l'exprime Lauriane (20 ans, portable avec cameraphone et APN, étudiante en $3^{\text {e }}$ année de licence) : « La fonction du portable change en fonction de l'appareil à côté ». Le cas de cette jeune étudiante semble paradigmatique. Résumons son histoire : ses parents lui ont offert il y a quelques années un APN à Noël qu'elle utilisait alors même qu'aujourd'hui elle se permet d'avoir un jugement critique à son égard :

« II était pas super, quand y a pas beaucoup de lumière, les photos elles sont atroces [...] Mais il était tout de même mieux que le portable que j'avais à l'époque, le premier qui faisait photo ».

Aussi, quand elle est amenée à changer de portable, elle prend très attentivement en compte le critère photo dans son achat, et c'est alors qu'elle utilise son portable (celui qu'elle a toujours au moment de l'enquête) pour faire des photos, délaissant quelque peu son APN. Ensuite, vient le temps de l'entrée à l'université, moment fort de sa vie puisqu'il correspond à la rencontre de nouvelles amitiés qui compteront pour elle (voir ses photos souvenirs des « repas de fin d'année universitaire » ou « des grèves »). Mais surtout, c'est la rencontre sur le campus avec son petit ami, rencontre qu'elle voudra immortaliser, puisque l'une de ses

\footnotetext{
18 Nous utiliserons désormais le sigle APN pour désigner l'appareil photo numérique.

19 Les APN compacts peuvent aussi devenir des objets incorporés, c'est le cas par exemple chez l'enquêtée Lauriane (voir infra).
} 
photos préférées sur portable n'était autre qu'un week-end avec ledit petit ami ${ }^{20}$ : « Mon premier week-end en amoureux avec mon chéri à Disneyland ». C'est alors qu'elle a l'idée d'offrir à ce petit ami un APN, qui réalise bien entendu de meilleures photos que son portable actuel.Aussi apparaît-il très vite que c'est elle qui va devenir l'utilisatrice principale de l'APN de son petit ami, n'oubliant jamais de lui rappeler de l'emporter quand ils partent ensemble à une soirée. Elle avoue utiliser alors de moins en moins son portable, pour des raisons essentiellement techniques influant sur la qualité de l'image : « Le problème, c'est que le portable, il a un temps de réaction, il met 5 secondes avant que l'image elle se stabilise, donc c'est dur d'avoir une photo nette ». Ses parents ayant finalement dû comprendre le message (elle n'utilise plus jamais l'APN qu'ils lui avaient offert à l'époque) lui ont en offert un nouveau au dernier Noël, évidemment plus performant techniquement (qualité de l'image, etc.) que tout ce qu'elle avait eu auparavant. Et l'on imagine aisément qu'elle a délaissé tous les autres dispositifs et n'utilise plus que celui-là : « Donc maintenant, je l'ai tout le temps sur moi ». C'est ainsi que cet APN compact est devenu un véritable objet incorporé, au même titre que le téléphone portable. Ce cas paradigmatique vise à illustrer combien se mettent en œuvre des rationalités d'usage qui ne peuvent être comprises qu'à la lumière des caractéristiques des autres appareils disponibles, mais aussi de I'histoire singulière, personnelle et affective, de l'usager. Après cette analyse de l'usage limité dans une perspective qualitative, nous allons tenter de comprendre ce même usage limité dans une perspective quantitative, afin de pouvoir ensuite réaliser une confrontation de ces deux approches complémentaires d'un même phénomène.

\section{L'usage limité : entre restrictions et volonté}

Selon la définition d'Everett Rogers, l'adoption est le choix d'une utilisation « complète » (« full use ») d'une innovation. Toutefois, une utilisation de toutes les fonctionnalités proposées sur un téléphone portable devient une hypothèse théorique qui ne se produit que dans un nombre très limité de cas. Dans notre échantillon, seulement $4 \%$ ont déjà utilisé au moins une fois les 9 fonctionnalités disponibles en plus de la téléphonie et des SMs : jouer, prendre et envoyer des photos, enregistrer et regarder des vidéos, écouter des mp3, aller sur l'internet, écouter la radio et regarder la télévision. La moitié des utilisateurs ( 195 pour la lère vague et 157 pour la $2^{\mathrm{e}}$ vague, donc 5 I \% de l'ensemble des usagers pour les deux vagues) ne se sont servis que de trois de ces fonctionnalités, soit un tiers

\footnotetext{
${ }^{20}$ Les photos immortalisant ce premier week-end auraient dû être prises avec l'APN, de meilleure qualité. Pourtant, elles l'ont été avec le portable, car l'APN n'avait alors pas été emporté à Disneyland, par peur de l'abimer ou le perdre dans les manèges. Où l'on voit que le jugement est relatif et sans cesse remis en question en fonction des nombreux critères définissant la situation (le critère est ici économique puisqu'il s'agit de préserver l'ApN ayant coûté plus cher que le portable).
} 
de ce qui est - à condition de disposer d'un appareil récent - techniquement possible (lllustration 4).

Illustration 3 : Pourcentage de participants qui utilisent entre une et neuf des fonctionnalités complémentaires du téléphone portable (moyenne des deux vagues, $N\left(\right.$ lère vague) $=346, N\left(2^{e}\right.$ vague $\left.)=342\right)$.

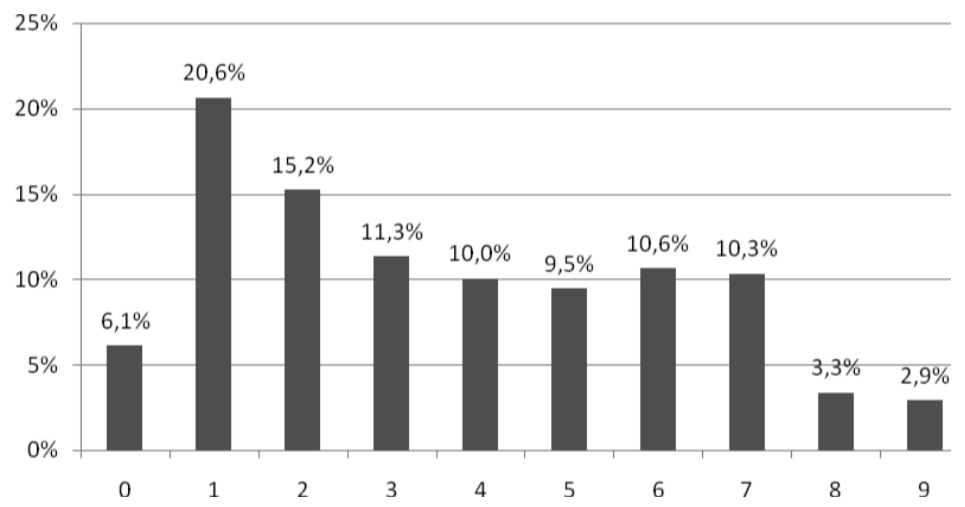

Ce constat permet à nouveau de distinguer entre deux groupes ceux qui utilisent une large variété des services et ceux qui se concentrent sur un nombre limité. Les usagers limités (qui utilisent au maximum 3 services) se concentrent sur les jeux (76\%), la prise de photos (37\%) et l'envoi de celles-ci (20\%). Les différences sont significatives pour toutes les fonctionnalités hormis les jeux (Illustration 5). Cette situation s'explique aisément du fait que les jeux sont disponibles sur tous les appareils, alors que les appareils photo ne sont intégrés que sur un nombre limité de téléphones.

Illustration 4 : Fonctionnalités utilisées par les usagers limités et les usagers intensifs - moyenne

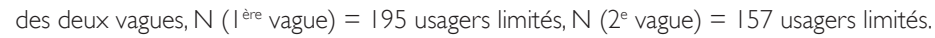

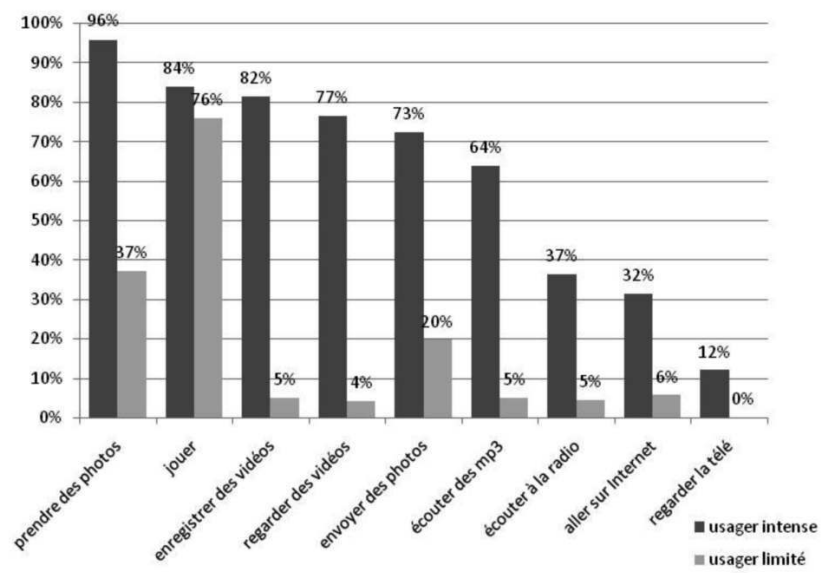


Dans un deuxième temps, comme au sein des non-usagers, on peut distinguer parmi les usagers qui se servent du téléphone de façon limitée des volontaires et des involontaires, c'est-à-dire ceux qui aimeraient bien utiliser plus de fonctionnalités mais sont freinés par des raisons extérieures et ceux qui choisissent librement de ne pas en utiliser plus. La base de cette distinction est une question sur les fonctionnalités que les participants souhaiteraient utiliser dans le futur. Ceux qui utilisent moins de quatre fonctionnalités aujourd'hui et ne cherchent pas en utiliser plus dans le futur sont considérés comme des utilisateurs limités volontaires, les autres comme des utilisateurs limités involontaires. Nous comptons dans la lère vague 119 usagers limités volontaires (61\%) et 76 usagers limités involontaires, pour la $2^{\mathrm{e}}$ vague, 103 usagers limités (66\%) volontaires et 54 usagers limités involontaires. À nouveau, on constate un moindre intérêt des usagers limités volontaires pour toutes les gratifications du téléphone portable. Pour eux, la sécurité est de loin la fonction la plus importante du téléphone portable. Les plus grands écarts avec les usagers intensifs et les usagers limités involontaires se trouvent dans la valeur de prestige et de divertissement, deux gratifications auxquelles les nombreuses fonctionnalités supplémentaires peuvent donner accès (Illustration 6) ${ }^{21}$. Le premier constat est que, en valeur absolue, les trois groupes considèrent pareillement l'importance de la sécurité. Ce constat ne surprend pas car la sécurité ne dépend pas des fonctionnalités supplémentaires : tout appareil permet d'appeler aux urgences gratuitement. Quant aux trois autres dimensions, on peut constater que les usagers limités volontaires y accordent moins d'importance que les involontaires. L'usage limité volontaire s'explique donc d'abord par un manque d'intérêt dans les gratifications qu'on pourrait tirer de la technologie.

Les parallèles entre les non-usagers volontaires et les usagers limités volontaires sont donc évidents: le comportement des deux s'explique moins par des restrictions parentales, financières ou techniques que par un manque d'intérêt dans les gratifications qu'on pourrait tirer de l'innovation. De plus, les usagers limités involontaires, comme les non-usagers involontaires, accordent plus d'importance à ces gratifications.

${ }^{21}$ Les différences entre les deux groupes d'usagers limités sont significatives pour la première vague au niveau du prestige $\left(T_{\text {vaguel }}=2,90 * *\right)$, du divertissement $\left(T_{\text {vaguel }}=4,47 * *\right.$ ) et de la gestion des relations $\left(T_{\text {vaguel }}=2,8 \mid * *\right)$ et pour la seconde vague au niveau du prestige $\left(T_{\text {vaguel }}=2,89 * *\right)$; celles entre usagers intensifs et l'ensemble des usagers limités pour les deux vagues au niveau du prestige $\left(T_{\text {vaguel }}=6,88 * * ; T_{\text {vague } 2}=3,60 * *\right)$, du divertissement $\left(T_{\text {vaguel }}=6,00 * * ; T_{\text {vague } 2}=3,83 * *\right)$ et de la gestion de relations $\left(T_{\text {vaguel }}=5,7 \mid * * ; T_{\text {vague } 2}=4,26 * *\right)$. 
Illustration 5 : Évaluation des gratifications par les usagers limités volontaires, les usagers limités involontaires et les usagers intensifs -1 =pas du tout important, $4=$ =très important; moyenne des deux vagues, $N\left(\left.\right|^{\text {ère }}\right.$ vague $)=320, N\left(2^{\text {e }}\right.$ vague $)=317$.

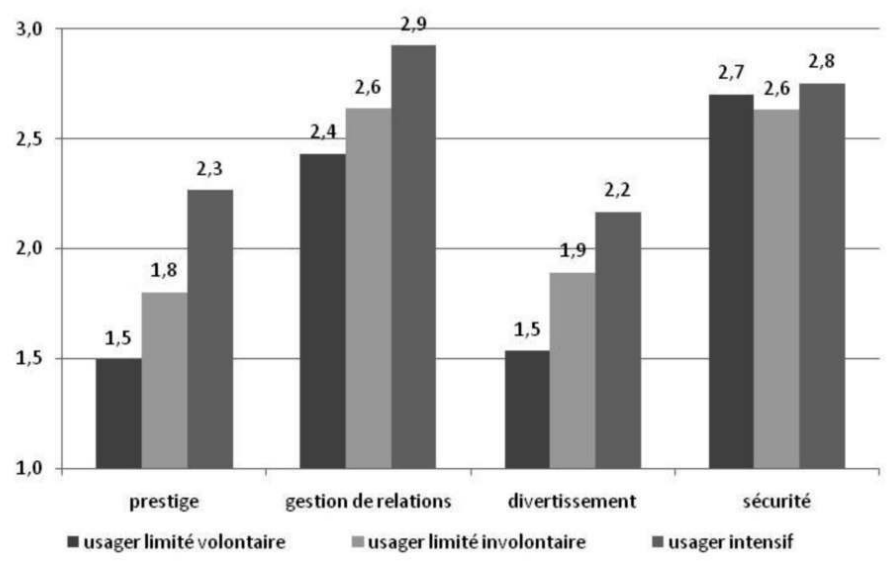

\section{Confrontation : un continuum usage/non-usage}

Grâce à la comparaison entre ces deux approches de l'usage limité/partiel, transparaît ce continuum usage/non-usage, au travers de l'arbitrage opéré par les individus eux-mêmes. Dans l'approche quantitative, l'attention est concentrée sur le média portable considéré comme un écosystème. L'objectif est de mettre en évidence et de décrire des patterns d'usage/non-usage des différentes fonctionnalités, subordonnés à l'appropriation de l'ensemble du dispositif que constitue le téléphone portable. Les individus opèrent donc des choix pour utiliser ou non telle ou telle fonctionnalité, en fonction des différentes gratifications (prestige, gestion de relations, sécurité, divertissement) qu'ils espèrent en retirer, des ressources et restrictions (notamment financières) et des normes sociales. Ces patterns représentent, de façon très sélective et fragmentaire, les arbitrages réalisés par les usagers entre les nombreux services proposés sur leur portable. Toutefois, il faut rappeler que ces résultats sont fondés sur des questions fermées et non des affirmations exprimées directement par les participants sur les perceptions qu'ils ont de leur usage/non-usage du téléphone. L'approche qualitative en apprend plus sur les logiques d'usage, notamment en élargissant l'attention au-delà du dispositif même du téléphone portable. Dans le raisonnement des usagers, l'usage d'autres dispositifs (appareil photo numérique, ordinateur) et l'entrelacement du téléphone avec d'autres médias permettent de comprendre les différents usages et non-usages. Face à l'offre pléthorique effectivement à la disposition des usagers, il apparait que ces derniers doivent opérer de véritables arbitrages pour orienter leur pratique. Ces arbitrages, qui 
aboutissent à la construction de compromis, résultent de l'analyse de critères techniques, économiques, mais aussi d'éléments plus personnels qui peuvent être identifiés grâce à la prise en compte de l'histoire singulière et subjective de chaque usager; et cela s'avère uniquement opérationalisable dans une approche qualitative.

\section{Conclusion}

L'intention n'était pas de nous engager dans une confrontation de type épistémologique qui opposerait les deux approches quantitative et qualitative. En revanche, la comparaison a permis d'apporter des éclairages complémentaires sur les nuances qui existent entre usage et non-usage et sur les raisons d'utiliser un téléphone portable de façon (très) limitée ou de ne pas l'utiliser du tout. L'hypothèse d'un continuum entre usage et non-usage s'est révélée fort pertinente. C'est ainsi que dans l'approche quantitative, les différences (en termes de gratifications attendues et en termes de normes) entre les deux catégories de non-usagers (volontaires et involontaires) sont, dans la majorité des cas, plus importantes que celles entre usagers et non-usagers. C'est pourquoi les nonusagers ne sauraient constituer un groupe homogène que l'on pourrait opposer de façon binaire aux usagers. Au contraire, nous avons découvert qu'un usage peut se limiter à très peu de fonctionnalités techniques si celles-ci correspondent à ce que les usagers attendent du téléphone portable et aux normes qui existent dans leur environnement proche. Le degré d'utilisation dépend aussi de l'offre technique en compétition avec le téléphone portable, comme par exemple un appareil photo numérique. L'usage et le non-usage s'expliquent alors de manière générale par un nombre de dimensions révélées par l'usager, son environnement social et l'offre technique; mais la question de savoir comment ces facteurs interagissent pour un usager spécifique ne peut être traitée dans une approche quantitative.

Notre vue différenciée sur les formes d'usages et non-usages permet aussi de mieux comprendre les non-usagers. Comme nous l'avons montré, leurs déficits (en termes de moyens financiers ou d'autorisations) ne jouent un rôle que dans les cas très spécifiques comme celui des jeunes adolescents encore dépendants de leurs parents. Hormis ces cas, les raisons pour un usage réduit ou un non-usage étaient soit conformes aux propres intérêts et aux normes de l'environnement immédiat, soit en vive opposition au sein d'une société perçue de façon très critique. Dans ce cas, une véritable continuité a été mise en évidence entre les légitimations d'usage et celles des non-usages puisque les non-usagers reprennent les légitimations des usagers pour les déconstruire dans un discours engagé de résistance face à une pratique sociale normative. Ce dernier exemple peut servir de base pour une réflexion sur la valeur ajoutée de l'approche qualitative et quantitative dans le cadre de nos questions de recherche et de 
nos échantillons : pour mieux comprendre le raisonnement des individus, c'est la méthode qualitative qui a fait avancer les connaissances; lorsqu'il était question d'identifier les facteurs (gratifications, normes, restrictions) qui influencent les différentes formes d'usages et non-usages, la méthode quantitative a permis d'évaluer leur importance respective. Ces constats correspondent d'ailleurs à ceux faits par d'autres sur la complémentarité des approches qualitatives et quantitatives (Verschuren, 200 I).

Le nombre limité des non-usagers dans les échantillons empêche d'aller au-delà de ces constats généraux et de généraliser les motivations spécifiques des nonusagers. Une autre limite importante de cette confrontation réside dans le fait que, bien que s'intéressant au même phénomène, ces deux enquêtes ne ciblent pas les mêmes publics, elles ont été réalisées à des époques différentes (2003/2004 pour l'enquête qualitative, 2006/2007 pour l'enquête quantitative) et elles sont issues de contextes culturels différents. Enfin, une dernière limite de taille réside dans le fait que la confrontation a été réalisée a posteriori, c'est-à-dire après la collecte des données. En effet, les deux enquêtes ont été conduites séparément, et c'est dans un second temps qu'une mise en perspective a été réalisée pour tenter de mettre en évidence des points de tension, de comparaison et de divergence. II semble évident qu'une autre perspective gagnerait à confronter les deux approches quantitatives et qualitatives au préalable, afin de les associer de manière véritablement complémentaire dans une seule et même enquête, et ce, dans le même contexte culturel et auprès du même public.

\section{Références}

Ajzen I., 1985, « From intentions to actions : a theory of planned behavior », pp. I I-39, in : Kuhl J., Beckmann J., dirs, Action-control : from cognition to behavior, Heidelberg, Springer.

Bakardjeva M., Smith R., 200I, «The Internet in Everyday Life. Computer Networking from the Standpoint of the Domestic User », New Media and Society, 3( I), pp. 67-83.

Bitkom (Bundesverband Informationswirtschaft, Telekommunikation und neue Medien e.V.), 2009, Datendienste geben dem Mobilfunk Schwung. Accès : https ://www.bitkom. org/files/documents/BITKOM_Presseinfo_EITO_TK-Markt_weltweit_09_02_2009_ final.pdf (Consulté le 3l/05/l0).

Blanchet A., Gotman A., 1992, L'enquête et ses méthodes. L'entretien, Paris, Nathan.

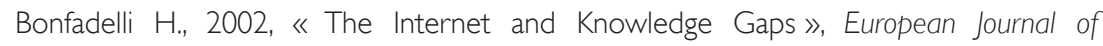
Communication, 17 ( I), pp. 65-84.

Breton Ph., Proulx S., 2002, L'explosion de la communication à l'aube du XXle siècle, Paris, Éd. La Découverte.

Bundesnetzagentur, 2009, Teilnehmerentwicklung im Mobiltelefondienst nach Netzen pro Quartal. Accès : : http ://www.bundesnetzagentur.de/cae/servlet/contentblob/I I 3850/ publicationFile/ I I 63/TeilnhmrEntwicklgPenetrtnld I 6875pdf.pdf. Consulté le 3 I/05/ I0. 
Caradec V., 200 I, « Personnes âgées et objets technologiques : une perspective en termes de logique d'usage », Revue française de sociologie, 42-1, pp. I 1 7- 148.

Castro-Thomasset E., 2000, L'apostasie de la télévision. Une forme d'iconoclasme contemporain, Paris, Éd. L'Harmattan.

Certeau de M., 1990, L'invention du quotidien. Tome I. Arts de faire, Paris, Gallimard, 1998.

Chambat P., 1994, « Usages des technologies de l'information et de la communication (TIC) : évolution des problématiques », Technologies de l'information et société, 6 (3), pp. 249-270.

Dearing J., Singhal A., 2006, Communication of Innovations, Thousand Oaks, Sage.

Delia J., 1987, « Communication Research : A History », pp. 20-98, in : Berger C., Chaffee S., dirs, Handbook of Communication Science, Newbury Park, Sage.

Dimmick J., Klein S., Stafford L., 2000, «The gratification niches of personal E-Mail and the telephone. Competition, Displacement and Complementarity », Communication Research, 27 (2), pp. 227-248.

Elliott P., 1974, « Uses and Gratifications Research: A Critique and A Sociological Alternative », pp. 249-268, in : Blumler J., Katz E., dirs, The Uses of Mass Communications, Beverly Hills, Sage.

Flichy P., 1992, « Présentation », Réseaux, « Les usages du téléphone », 55, sept.-oct.

- 1995, L'innovation technique. Récents développements en sciences sociales vers une nouvelle théorie de l'innovation, Paris, Éd. La Découverte.

— 1997, « Perspectives pour une sociologie du téléphone », Réseaux, 82/83, mars-juin, pp. 7-20.

— 2008, «Technique, usage et représentations 》, Réseaux, vol. 2., I 48//49, pp. 147- 174.

Gaglio G., 2005, « Pour un regard tempéré sur les « réfractaires » aux biens massivement diffusés. Variations autour du cas de la téléphonie mobile en France », Réseaux, vol. 5, 133, pp. 167-198.

- 2008, « La dynamique des normes de consommation : le cas de l'avènement de la téléphonie mobile en France », Réseaux, vol. 2, 2, pp. 181-198.

Goffman I., 1974, Les cadres de l'expérience, trad. de l'anglais par I. Joseph, M. Dartevelle et P. Joseph, Paris, Éd. de Minuit, 1991.

Haddon L., 2003, « Domestication and Mobile Telephony », pp. 43-46, in : Katz J., dir., Machines That Become Us: The Social Context of Personal Communication Technology, New Brunswick, Transaction Publishers.

Hall S., 1980, « Encoding/Decoding », pp. 128-1 38, in : Hall S., Hobson D., Lowe A., Willis P., dirs, Culture, Media, Language, London, Hutchinson.

Hampton K., Livio O., Sessions L., «The Social Life ofWireless Urban Spaces : Internet Use, Social Networks, and the Public Realm », Journal of Communication, à paraitre.

Hargittai E., 2002, « Second-Level Digital Divide : Differences in People's Online Skills » First Monday, 7 (4). Accès : http ://firstmonday.org/htbin/cgiwrap/bin/ojs/index.php/fm/ article/view/942/864. Consulté le 31/05/I 0.

Holmes D., Russel G., 1999, « Adolescent CIT use : paradigm shift for educational and cultural practices? », British Journal of Sociology of Education, 20 (I), pp .69-78. 
Non-usages du téléphone portable

Hung S., Ku C., Chan C., 2003, « Critical factors of WAP services adoption : an empirical study » Electronic Commerce Research and Applications, 2 ( I ), pp. 42-60.

Jarrigeon A., Menrath J., dirs, 2007, Le téléphone mobile aujourd'hui. Usages et comportements sociaux, $2^{\mathrm{e}}$ éd., rapport de recherche. Accès : www.afom.fr.

Jauréguiberry Fr., 2003, Les branchés du portable, Paris, Presses universitaires de France.

— 2007, « Les téléphones portables, outils du dédoublement et de la densification du temps : un diagnostic confirmé », Tic \& société, vol. I, I. Accès : www.revue-tis.org.

Jouët J., 1993, « Pratiques et figures de la médiation », Réseaux, 60, pp. 99- 120.

— 2000, «Retour critique sur la sociologie des usages », Réseaux, 100, pp. 487-521.

Julien M.-P., Warnier J.-P., 1999, Approches de la culture matérielle. Corps à corps avec l'objet, Paris, Éd. L'Harmattan.

Karnowski V., von Pape T., Wirth W., 2008, « After the digital divide? An appropriation perspective on the generational mobile-phone divide », pp. 177-193, in : Hartmann M., Rössler P., Höflich J., dirs, After the Mobile Phone? Social Changes and the Development of Mobile Communication, Berlin, Frank \& Timme.

Katz E., Blumler J., Gurevitch M., 1974, « Ulilization of mass communication by the individual », pp. 19-32, in : Blumler J., Katz E., dirs, The uses of mass communications : Current perspectives on gratifications research, Beverly Hills, Sage.

Katz E., Foulkes D., 1962, « On the uses of mass media as "escape": clarification of a concept », Public Opinion Quarterly, 26 (3), pp. 377-388.

Kincaid D., 2004, « From Innovation to Social Norm : Bounded Normative Influence », Journal of Health Communication, 9, pp. 37-57.

Lazarsfeld P., Reuben B., Gaudet H., 1944, The people's choice : How the voter makes up his mind in a presidential campaign, New York, Duell, Sloan, and Pearce.

Lehtonen, T., 2003, «The Domestication of New Technologies as a Set of Trials », Journal of Consumer Culture, 3 (3), pp. 363-385.

Leung, L., 2000, « More than just talk on the move : uses and gratifications of the cellular phone », Journalism and Mass Media Quarterly, 77 (2), pp. 308-320.

Licoppe C., 2002, « Sociabilité et technologies de communication. Deux modalités d'entretien des liens interpersonnels dans le contexte du déploiement des dispositifs de communication mobiles », Réseaux, vol. 20, I 12-1 13, pp. 171-210.

Ling R., 1999, «I am happiest by having the best: The adoption and rejection of mobile telephony », R\&D report 15/99, Kjeller, Telenor. Accès: http ://www.richardling.com/ papers/1999_happiest_having_the_best.pdf. Consulté le 31/05/10.

Ling R., Nilsen S., Granhaug S., 1999, «The domestication of video-on-demand: folk understanding of a new technology », New Media and Society, I (I), pp. 8I- I00.

Livingstone S., Helsper E., 2007, « Gradations in digital inclusion : children, young people and the digital divide », New Media and Society, 9 (4), pp. 67।-696.

Mallein P., Toussaint Y., 1994, « L'intégration sociale des technologies d'information et de communication: une sociologie des usages », Technologies de l'information et société, vol. 6, 4, pp. 315-335. 
Martin C., 2007a, « Le téléphone portable, un objet incorporé? », pp. 79-90, in : Manoha M., dir., In-corporer, Paris, Éd. L'Harmattan.

- 2007b, Le téléphone portable et nous. En famille, entre amis, au travail, Paris, Éd. L'Harmattan.

- 2008, « Téléphone portable et paraître. De nouvelles normes sociales chez les adolescents? », pp. ||5-130, in: Paresys I. (éd.), Paraître et apparences en Europe occidentale du Moyen Âge à nos jours, Villeneuve d'Ascq, Presses universitaires du Septentrion.

— 2010, « Le téléphone portable : des légitimations des usages à celles des non-usages 》, pp. 181-195, in : Belot R., Heyberger L., éds, Prométhée et son double. Craintes, peurs et réserves face à la technologie, Neuchâtel/Toulouse, Alphil-Presses universitaires suisses/ Éd. Méridiennes.

McQuail D., 2005, McQuail's mass communication theory, London, Sage.

Paquienséguy, 2007, « Comment réfléchir à la formation des usages liés aux technologies de l'information et de la communication numériques? »», Les Enjeux de l'information et de la communication. Accès : http ://w3.u-grenoble3.fr/les_enjeux/2007/Paquienseguy/ index.php. Consulté le 25/02/10.

Perriault J., 1989, La logique de l'usage. Essai sur les machines à communiquer, Paris, Flammarion.

Popper K., 1973, La logique de la découverte scientifique, trad. de l'allemand par N.ThyssenRutten, P. Devaux, Paris, Payot, 2007.

Pronovost G., 1994, « Médias : éléments pour l'étude de la formation des usages sociaux », Technologies de l'information et société, 6 (4), pp. 337-400.

Quandt T., von Pape T., 2010 « Living in the mediatope: A multi-method study on the evolution of media technologies in the domestic environment », The Information Society, 26 (à paraître).

Réseaux, 2002, « Mobiles », vol. 20, I | 2- II 3 .

—, 2009, « Les usages avancés du téléphone mobile », vol. 27, 156.

Renaud K., van Biljon J., 2008, « Predicting technology acceptance and adoption by the elderly: a qualitative study », ACM International Conference Proceeding Series, vol. 338, pp. $210-219$.

Rivière C.-A., 2002, « La pratique du mini-message. Une double stratégie d'extériorisation et de retrait de l'intimité dans les interactions quotidiennes », Réseaux, vol. 20, | | 2- | | 3, pp. $139-168$.

Robinson J., DiMaggio P., Hargittai E., 2003, « New social survey perspectives on the Digital Divide », Information Technology and Society, I (5), pp. I-22.

Rogers E., 1962, Diffusion of Innovations, New York, Free Press, 2003.

— 2000, «The digital divide », Convergence, 7 (4), pp. 96- I I I.

Rogers E., Kincaid L., 1981, Communication Networks :Toward a New Paradigm for Research, New York, McMillan.

RuggieroT., 2000, «Uses and gratifications theory in the $21^{\text {st }}$ century » Mass Communication \& Society, 3 ( I), pp. 3-37. 
Non-usages du téléphone portable

Schenk M., Dahm H., Sonje D., 1997, « Die Bedeutung sozialer Netzwerke bei der Diffusion neuer Kommunikationstechniken », Kölner Zeitschrift für Soziologie und Sozialpsychologie, 49 ( I), pp. 35-52

Selwyn N., 2003, «Apart from technology : understanding people's non-use of information and communication technologies in everyday life », Technology in Society, 25 ( I), pp. 99-। 16.

Sherry J., Lucas K., Greenberg B, Lachlan K., 2006, « Video game uses and gratifications as predictors of use and game preference », pp. 21 3-224, in :Vorderer P., Bryant J., dirs, Playing video games : Motives, responses, and consequences, Mahwaw, Lawrence Erlbaum Associates.

Silverstone R., Haddon, L., 1996, «Design and the domestication of information and communication technologies: Technical change and everyday life », pp. 44-74, in : Silverstone R., Mansell R., dirs, Communication by Design. The Politics of Information and Communication Technologies, Oxford, Oxford University Press.

Teo T., Pok S., 2003, « Adoption of WAP-enabled mobile phones among Internet users », Omega, 31, pp. 483-498.

Trepte S., Ranné N., Becker M., 2003, «"Personal Digital Assistants » - Patterns of user gratifications », Communications. European Journal of Communication Research, 8 (4), pp. 457-473.

Valente T., 2006, « Communication Network Analysis and the Diffusion of Innovations », pp. 6I-82, in : Singhal A., Dearing J., dirs, Communication of Innovations : a journey with Ev Rogers, New Delhi, Sage.

Van DijkJ., Hacker K., 2003, «The Digital Divide as a Complex and Dynamic Phenomenon », The Information Society 19 (4), pp. 315-236.

Verschuren P., 200I, « Holism versus reductionism in modern social science », Quality \& Quantity, 35, pp. 389-405.

Von Pape T., 2008, Aneignung neuer Kommunikationstechnologien in sozialen Netzwerken, Wiesbaden, VS Research.

— 2009, « Media adoption and diffusion. », pp. 274-282, in : Hartmann T., dir., Media Choice. A theoretical and empirical overview, New York \& London, Routledge.

Von Pape T., KarnowskiV., Wirth W., 2006, « La fracture numérique dans nos mains : Enjeux sociaux de l'appropriation du téléphone portable par un groupe d'adolescents », pp. 73-83, in: Patteson R., dir., Enjeux et usages des TIC. Reliance sociale \& insertion professionnelle, Bruxelles, Éd. CREATIC.

— 2008, « Eine integrative Skala zur Messung des Konstruktes der Aneignung », pp. 96- 127 , in : Matthes J., Wirth W., Fahr A., Daschmann G., dirs., Die Brücke zwischen Theorie und Empirie : Operationalisierung, Messung und Validierung in der Kommunikationswissenschaft, Cologne, Herbert von Halem.

Wei R., Lo V., 2006, « Staying connected while on the move : Cell phone use and social connectedness », New Media and Society, 8 ( I ), 53-72. 
$-1$

$1_{-}^{1}$

$-1$

$1^{-}$ 